Nota Editorial

\section{LOS ESTUDIOS LATINOAMERICANOS DE REDUCCIÓN DEL RIESGO DE DESASTRES EN EL CONTEXTO DE LA PANDEMIA DEL COVID-19}

Adriana Allen $^{1,2^{*}}$, Juan Pablo Sarmiento ${ }^{3}$, Vicente Sandoval ${ }^{4}$

\section{RESUMEN}

Prácticamente ningún gobierno, institución o comunidad ha permanecido indiferente a la pandemia del COVID-19. Esta crisis ha sido señalada en muchos lugares como un 'desastre', por lo que se ha considerado apropiado hacer una breve reflexión sobre el significado y lecciones por aprender de esta pandemia en el contexto de los estudios sobre reducción y prevención del riesgo de desastres en Latinoamérica. Los autores sostienen que la pandemia hace visible las desigualdades estructurales que dan forma a lo que significa ser afectado por el virus. Resaltan cinco aspectos que merecen atención: el sub-registro de los impactos de la crisis, la importancia de diferenciar entre medidas de protección 'similar' y medidas de protección 'igualitaria', el cómo prevenir la profundización de procesos de estigmatización, la adopción de un enfoque de derechos humanos al hábitat en el diseño e implementación de respuestas, y la urgencia de actuar haciendo frente a los desafíos postergados del desarrollo. Por último, los autores reflexionan sobre los marcos interpretativos existentes en la gestión de emergencias de salud pública y en la de riesgos socio-naturales, y cómo éstos pueden orientar procesos de investigación que permitan una mejor comprensión de los factores del riesgo a modo de informar decisiones transformativas.

\section{PALABRAS CLAVE}

COVID-19, Reducción del Riesgo de Desastre, América Latina, Derechos al hábitat, Trampas de riesgo

\section{LATIN AMERICAN STUDIES ON DISASTER RISK REDUCTION IN THE CONTEXT OF THE COVID-19 PANDEMIC}

\section{ABSTRACT}

Practically no government, institution or community has remained indifferent to the COVID-19 pandemic. This crisis has been pointed out in many places as a 'disaster'. For this reason, REDER has considered appropriate to dedicate a brief but necessary reflection on the meaning of and lessons to be learned from this pandemic in the context of Latin American studies on disaster risk reduction and prevention. The authors argue that the pandemic has exposed the structural inequalities that shape the uneven impacts among different social groups. According to the authors, five aspects need to be highlighted: the under-recorded impacts of the crisis; differentiation between 'similar' protection measures from 'egalitarian' protection; how to prevent stigmatization; design and implementation of responses based on a human rights approach to habitat; and time to act addressing the postponed development challenges. Finally, the authors look at the differences between the existing interpretative frameworks in the management of public health emergencies and in that of socio-natural risks. These could guide research processes that enable a better understanding of the dynamics and risk factors in the future, and therefore inform transformative decision-making.

\section{KEYWORDS}

COVID-19, Disaster Risk Reduction, Latin America, Habitat rights, Risk traps
1. The Bartlett Development Planning Unit, University College London, Londres, Reino Unido.

2. Coalición Internacional del Hábitat (HIC), Londres, Reino Unido.

3. Extreme Events Institute, Florida International University, Miami, FL, EEUU.

4. Disaster Research Unit, Freie Universität Berlin, Alemania.

*Autor de correspondencia: a.allen@ucl.ac.uk

\section{RECIBIDO}

23 de marzo de 2020

\section{PUBLICADO}

1 de julio de 2020

\section{Formato cita}

Recomendada (APA): Allen, A., Sarmiento, J.P. \& Sandoval, V. (2020). Los Estudios Latinoamericanos de Reducción del Riesgo de Desastres en el Contexto de la Pandemia del COVID-19. Revista de Estudios Latinoamericanos sobre Reducción del Riesgo de Desastres REDER, 4(2), 1-6. https://doi.org/10.55467/ reder.v4i2. 46

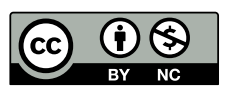

Todos los artículos publicados en REDER siguen una política de Acceso

Abierto y se respaldan en una Licencia CreativeCommons Atribución-NoComercial 4.0 Internacional.

Revista de Estudios Latinoamericanos sobre Reducción del Riesgo de Desastres (REDER)

Diseño: Lupe Bezzina Tipografía: Hospital 


\section{CONTEXTO DE LA PANDEMIA DEL COVID-19 EN LA REGIÓN}

La situación que antecede a la publicación de este número de la revista REDER, en Julio de 2020, es sumamente inusual, por calificarla de algún modo. Prácticamente nadie, ningún gobierno, institución, empresa o asociación comunitaria -tanto en la región como a nivel global- ha permanecido indiferente a la pandemia del COVID-19, enfermedad causada por el virus SARSCoV-2, detectado inicialmente en diciembre de 2019 en la ciudad de Wuhan, China. Esta pandemia ha sido señalada en muchos lugares como una 'catástrofe' o 'desastre', generalmente al tiempo que las cifras comienzan a ser amenazadoras: números de contagios y muertes, escasez de camas de cuidados intensivos y ventiladores mecánicos, y personal sanitario infectado, entre otros. Por este motivo, consideramos apropiado hacer una breve pero necesaria reflexión sobre el significado y lecciones por aprender de esta pandemia en el contexto de los estudios sobre reducción del riesgo de desastres (RRD) en Latinoamérica, en conjunto con los artículos regulares de este número.

Al momento en que se escribe este texto, todos los países de la región se encuentran luchando contra la pandemia. Actualmente, el país latinoamericano más afectado por este nuevo coronavirus es Brasil, con más de 1,4 millones de casos y 60 mil muertes. Hasta el 2 de julio de 2020, la región ha registrado más de 2,37 millones de personas infectadas, así como un número creciente de fatalidades. Sin aventurar un análisis prematuro sobre cómo se está enfrentando la pandemia a nivel regional, es claro que la pandemia continúa desarrollándose día a día, con avances y retrocesos, en cada país, territorio y comunidad. La actual crisis sanitaria, amenaza con transformarse en un 'desastre', dependiendo del nivel de exposición, vulnerabilidad y capacidad de acción en diferentes contextos. Unas semanas después del inicio de la epidemia fue posible identificar algunos patrones donde se evidenció que su impacto recaía más severamente en aquellos más marginados, aquellos que, por causas directas o indirectas, han sido 'expulsados' del sistema formal de bienestar: vivienda, suelo, transporte y acceso a oportunidades, salud y educación, entre otros.

No debemos olvidar que, antes de que llegara la pandemia a la región, ya había unos 105 millones de personas viviendo en asentamientos precarios, con acceso limitado a servicios básicos y en muy malas condiciones habitacionales, esto es, que 1 de cada 5 hombres y mujeres de la región estaban viviendo en condiciones precarias (Sandoval \& Sarmiento, 2020).

En el mundo académico de nuestra área de estudio, no han sido pocos los autores que han intentado aplicar los marcos conceptuales de este campo específico al contexto de esta pandemia. Algunos, como Lavell et al. (2020), han ofrecido una lectura fresca y novedosa de la crisis, aplicando experiencias y conocimientos acumulados por décadas en los estudios sociales del riesgo de desastre: miradas desde la 'sociología de los desastres', o desde la 'economía política' y la 'vulnerabilidad' entre otras, aportan así nuevas perspectivas. Por su parte, Allen (2020) nos invita a considerar cómo y por qué la pandemia es solo una entre muchas otras condiciones de riesgo que afectan en forma desproporcional a los sectores populares. Otras, como Wilkinson (2020), ofrecen consideraciones prácticas para abordar la pandemia de COVID-19 en asentamientos urbanos precarios, y con ello reducir el riesgo de impactos mayores. Hay algunos incluso, que han considerado al contexto de la pandemia y sus impactos como un 'desastre lento' (Gonzalez et al., 2020) o de gradual maduración, esto es exacerbando los ciclos de acumulación de riesgo que Allen et al. (2018) denominan 'trampas de riesgo urbano'.

Nos interesa, por lo tanto, aprovechar la oportunidad de este nuevo número para subrayar algunos temas que consideramos de suma importancia integrar tanto al análisis y aprendizaje como en las respuestas actuales y futuras a la pandemia del COVID-19 en la región, sea desde comunidades locales, gobiernos locales y nacionales, u organismos que trabajan de forma bilateral e internacional.

\section{RIESGO Y PANDEMIA: ENTRE LO COTIDIANO Y LO EXCEPCIONAL}

En menos de un mes, y siguiendo el ejemplo de China, a nivel internacional, la mayoría de los gobiernos han adoptado enfoques similares para hacer frente a la pandemia de COVID-19, imponiendo primordialmente medidas de cuarentena, aislamiento y distanciamiento físico. Las medidas aplicadas en muchos países de América Latina han seguido un camino similar y son cada vez más estrictas. Pero muchas de estas medidas de protección -aunque necesarias- son impracticables para un gran número de ciudadanas y ciudadanos o incluso plantean más riesgos. 
El mensaje es claro: la adopción de premisas inmunológicas generalizantes no funciona para muchas y muchos.

Para la mayoría de la gente que vive en asentamientos informales, quedarse en casa no es una opción, ni una medida de protección, sino un riesgo adicional. Esto se agrava por el riesgo de exposición que supone hacer cola para usar los baños compartidos, sacar agua de pozos o perforaciones, o usar el abarrotado transporte público. El gran número de mujeres, hombres, niñas y niños que viven en asentamientos informales se verán perjudicados por medidas que asumen que el hogar es un paraíso seguro, que las personas pueden almacenar alimentos, echar mano a sus ahorros o trabajar desde casa.

EI COVID-19 hace visible cómo las profundas desigualdades estructurales dan forma a lo que significa ser afectado por el virus no sólo a través de diferentes geografías sino también a través de la intersección de clases, razas, géneros, edades y habilidades. En las últimas semanas, hemos leído relatos devastadores de cómo las políticas de bloqueo obligaron a muchos habitantes urbanos a elegir entre ingresos y seguridad, entre hambre y enfermedad, entre salud y medios de vida. Correr riesgos forma parte de su vida cotidiana. Esta pandemia también pone de relieve que los gobiernos nacionales y el sector del desarrollo son muy reacios a correr riesgos. Los donantes prestan apoyo basándose en la certeza de la entrega de productos y resultados predefinidos. El hecho de no cumplir con las condiciones establecidas suele significar la pérdida del apoyo. Esto debe cambiar, ya que este enfoque ofrece poco margen para el aprendizaje práctico.

Entonces, ¿qué podemos cambiar para asegurar que la conmoción que se siente hoy en día en todo el mundo abra camino para los cambios estructurales que se necesitan desde hace mucho tiempo?

En primer lugar, tenemos que poner números a los impactos poco registrados de la crisis. Todos los días nos desper tamos con nuevas actualizaciones sobre el número de personas infectadas y las vidas cobradas por el coronavirus en todo el mundo. Pero rara vez vemos cifras en los medios de comunicación sobre el número de personas que se verán afectadas desproporcionadamente debido a las desigualdades preexistentes. Sabemos del incremento persistente de personas viviendo en asentamientos informales a nivel mundial. Asimismo, somos conscientes de los vergonzosos déficits mundiales que siguen impidiendo a muchas y muchos acceder al agua potable y al saneamiento. Pero debemos seguir desagregando estas cifras, ponerlas en la mirada pública y de nuestros gobiernos para exigir el nivel de recursos necesarios para garantizar que se aborde de una vez por todas la atención estructural a desigualdades profundamente arraigadas.

En segundo lugar, debemos reconocer que medidas de protección 'similares' no significan protección 'igualitaria'. Las medidas generales que tratan de abordar en forma universal la realidad de diferentes tipos de asentamientos informales serán inevitablemente ineficaces. Una barriada peruana es muy diferente a un barrio popular en un contexto semi-rural en Malawi. Un asentamiento informal en Freetown (Sierra Leona) es diferente a un campamento de refugiados en muchas ciudades europeas. Por evidente que esto pueda parecer, cada uno de estos ámbitos demanda respuestas contextualizadas. Asimismo, es importante recordar que las mujeres, niñas, niños y ancianos son desproporcionadamente afectadas por la crisis del COVID-19. Las mujeres representan el $70 \%$ de la fuerza de trabajo del sector sanitario y de trabajo social a nivel mundial, la crisis actual las pone en mayor riesgo y requiere una atención especial a su salud y necesidades.

En tercer lugar, es imprescindible prevenir la estigmatización. La sensibilización en los asentamientos informales sobre cómo evitar la infección, qué hacer si se está infectado y cómo cuidar a los demás, son tareas fundamentales en contextos en los que la capacidad de asistencia sanitaria pública es débil o está en gran medida ausente. Si bien el mensaje de lavarse las manos es universalmente pertinente -al menos en los lugares donde las personas pueden acceder al agua y al jabón-, hay otros que deben ser considerados cuidadosamente para evitar una mayor discriminación. Sólo vale recordar los primeros mensajes sobre el HIV o SIDA en África que crearon un enorme estigma en torno a aquellos y aquellas susceptibles a ser afectados por el virus. Esto hizo que muchas personas se sintieran reacias a someterse a las pruebas y ayudó a que la enfermedad se propagara más rápidamente.

En cuarto lugar, la protección de los derechos humanos debe ser el foco primordial de las respuestas al COVID-19, tanto durante como después de la pandemia. Las cuarentenas en Ruanda y la India han visto ciudadanos muertos por la policía por romper el toque de queda. Debemos 
recordar que las mujeres y los hombres que viven en asentamientos informales suelen tener una relación problemática con las instituciones estatales, condición que se extiende al uso de desalojos forzados, justificados como medidas de 'protección social'.

Por último, pero no por ello menos importante, es hora de actuar para hacer frente a los desafíos que se deben abordar desde hace mucho tiempo. Para la mayoría de las mujeres y hombres, ancianas y ancianos, niñas y niños que viven en asentamientos informales, el Covid-19 será sólo una de las muchas amenazas para la salud que ellas y ellos enfrentarán a lo largo de sus vidas. En este contexto, es fundamental presionar a los gobiernos y a la comunidad internacional para poner fin a algo que no atiende a razones: La falta de sentido de los desalojos forzosos, como medio para atacar el hacinamiento y proteger los asentamientos precarios; la falta de sentido de mercados de tierra y vivienda que empujan a muchas y muchos a vivir en entornos ambientalmente marginales y en condiciones de gran inseguridad de tenencia. La falta de sentido de vivir en un mundo en el que más del $11 \%$ de la población sigue careciendo de suministro adecuado de agua y un $25 \%$ sin acceso a un saneamiento adecuado. La falta de sentido de cadenas alimenticias derrochadoras y mercantilizadas que condenan a la gente a la inanición y a casi el $11 \%$ de la población mundial a la desnutrición crónica. La falta de sentido de ignorar la desigualdad de género en todas las esferas de la vida, y la lista continúa.

El desafío principal al que nos enfrentamos no consiste sólo en poner en marcha medidas de protección sanitaria durante esta crisis, sino en hacer frente a las profundas desigualdades que existen ahora y más allá de la pandemia. Los Objetivos mundiales hacia el Desarrollo Sustentable (ODSs) ya explican los desafíos interconectados que deben abordarse y han sido aprobados por 193 países. Pero es claro que no podemos esperar hasta 2030 para convertirlos en realidad. El derecho a la ciudad es el derecho a la vida. Esto significa reconocer que la producción social del hábitat es también la producción social de la salud.

\section{MARCOS INTERPRETATIVOS DE GESTIÓN DE EMERGENCIAS DE SALUD PÚBLICA Y DE RIESGOS SOCIO-NATURALES}

Una de las lecciones por aprender de la crisis del COVID-19 consiste en que las políticas y mecanismos dispuestos para la gestión del riesgo y el manejo de la emergencia ante eventos de origen socio-natural y antrópico fueron completamente superados, y jugaron un papel secundario ante el evento, por su magnitud y extensión geográfica sin precedentes.

Conviene reflexionar entonces sobre cuáles han sido los factores que hacen de esta crisis un hecho sin precedentes recientes. Para ello es necesario iniciar contrastando los marcos interpretativos que hay detrás de la gestión de las emergencias de salud pública asociadas con las amenazas biológicas, y la gestión de riesgos socio-naturales. En la primera predomina el enfoque ecológico de los riesgos para la salud, mientras para la segunda impera el enfoque sistémico, basándose en conceptos de amenaza, exposición, vulnerabilidad y riesgo (Sarmiento, 2015). Hay convergencias y especificidades en los dos enfoques, de las cuales se derivan políticas, estrategias y aproximaciones diferentes. Estos marcos deben también ser tenidos en cuenta al momento de avanzar en procesos de investigación que permitan aportar a una mejor comprensión de las dinámicas y factores involucrados a modo de informar la toma de decisiones.

Trascendiendo las diferencias de los marcos de referencia, podemos emplear instrumentos interpretativos de la gestión de riesgos a las emergencias de salud pública como el COVID-19. En este sentido podemos indicar que la pandemia ilustra simultáneamente al riesgo 'intensivo' como al 'extensivo'. En el caso del riesgo intensivo, por un dramático incremento de la morbilidad y mortalidad en áreas específicas en días o semanas. En cuanto al extensivo, por la amplia dispersión a través de países, regiones y continentes, en donde se aprecia un proceso acumulativo en términos de pérdidas de vidas, de la sobrecarga a los sistemas de salud, y del impacto socioeconómico de las medidas tomadas para contener la pandemia.

Empleando el enfoque sistémico para evaluar estos riesgos biológicos que afectan la salud humana debemos proceder primero a la caracterización de los riesgos biológicos. Estos incluyen aspectos tales como infectividad, patogenicidad y virulencia, el factor de reproducción $R_{0} 0$ potencial de propagación, dosis infecciosas y supervivencia fuera del huésped. Luego, analizamos la exposición empleando criterios tales como factores del huésped, factores ambientales, transmisión, reservorios y vectores que lo transmiten. Finalmente, la vulnerabilidad, un campo explorado 
exhaustivamente en la salud pública, que se caracteriza por factores como las características de la población y la infraestructura física y social. Estos factores se desglosan aún más en los llamados 'determinantes sociales de la salud': (a) entorno social y económico -educación, servicios de salud, redes de apoyo social; apoyo de familias, amigos y comunidades, cultura, costumbres, tradiciones, creencias, ingresos y estatus social; (b) entorno físico -calidad de agua y aire; seguridad en el lugar de trabajo, la casa, la comunidad y los caminos; todos contribuyen a la buena salud, el empleo y las condiciones de trabajo; y (c) las características individuales de la persona -comportamientos, genética y habilidades de afrontamiento.

La complejidad de la medición y la interacción de los tres factores de riesgo (amenazas, exposición y vulnerabilidad) se reflejan a su vez en la complejidad de los modelos utilizados para evaluar el riesgo sistémico para la salud de los peligros biológicos, modelos que además incluyen las políticas, estrategias y tácticas necesarias para manejarlos, lo que se traducen en capacidades y recursos.

Si bien en las primeras semanas de la expansión de la pandemia a nivel mundial el COVID-19 incidió particularmente en un segmento de población de ingresos medios y altos, con capacidad de movilización internacional, niveles políticos, sociales y económicos. En las etapas posteriores de la pandemia, el impacto retomó las tendencias observadas en otras emergencias y desastres, caracterizado por el contagio comunitario, con un desproporcionado impacto socioeconómico en grupos vulnerables: migrantes, refugiados, poblaciones desplazadas y residentes de asentamientos informales y de alta densidad. Grupos que en situaciones regulares están al margen o carecen de servicios sociales y de salud.

Las medidas de aislamiento, distanciamiento físico, protección e higiene tomadas nos han ayudado a disminuir la incidencia y la dispersión de la enfermedad, manteniendo la demanda de asistencia en salud dentro de las capacidades existentes, pero el riesgo de reintroducción y resurgimiento de la enfermedad continuará y deberá controlarse de manera sostenible mediante la aplicación rigurosa de intervenciones de salud pública. Es muy probable que el virus circule entre y dentro de los países por un periodo de meses o incluso años. Será el desarrollo y la disponibilidad de vacunas, y las terapias seguras y efectivas lo que pueda permitir alcanzar una solución definitiva y a largo plazo.

Mientras tanto, enfrentaremos el dilema sobre la continuidad y rigor de las medidas de aislamiento y la severidad de su impacto socioeconómico.

Conviene recordar que a pesar de que nuestra atención está centrada en la pandemia, es necesario asegurar la continuidad de los programas regulares de prevención, promoción y atención en salud, así como la gestión del riesgo y el manejo de emergencias y desastres asociados a otros fenómenos socio-naturales como sismos, huracanes, inundaciones, que puedan ocurrir en forma concomitante durante esta pandemia.

Finalmente, es importante recalcar que somos conscientes de que en los próximos meses y años surgirán nuevas perspectivas sobre esta crisis que emergerán desde diferentes disciplinas, e incluso desde áreas multi- o transdisciplinarias, que busquen ser un aporte a la reducción del riesgo de desastre en el futuro. Con eso en la mira, las reflexiones ofrecidas en esta nota editorial intentan ser un apoyo a la agenda de investigadores e investigadoras interesadas en estudiar este fenómeno en la región.

\section{REFERENCIAS}

Allen, A. (2020, May 4). Beyond immunological assumptions. Video Presentation for the Global Platform for the Right to the City $\left(\mathrm{GPR}_{2} \mathrm{C}\right)$ First Assembly on Habitat Rights under COVID-19 [Video]. Habitat International Coalition's Youtube Channel. https://youtu.be/ElBnNKtVo-M

Allen, A., Zilbert Soto, L., Wesely, J., Belkow, T., Ferro, V., Lambert, R., Langdown, I., \& Samanamú, A. (2018). From state agencies to ordinary citizens: Reframing risk-mitigation investments and their impact to disrupt urban risk traps in Lima, Peru. Environment and Urbanization, 29(2), 477-502. https://doi.org/10.1177/0956247817706061

Gonzalez, R., Bacigalupe, G., Farias, C., \& Sandoval, V. (2020). COVID-19 y la ilusión de que podemos frenar un tren de carga en pocos metros. Centro de Investigación Periodística CIPER - Chile. http:// bit.ly/Tren-COVID19 
Lavell, A., Mansilla, E., Maskrey, A., \& Ramirez, F. (2020). La construcción social de la pandemia COVID-19: Desastre, acumulación de riesgos y políticas públicas (pp. 1-10). La RED. https://www. desenredando.org

Sandoval, V., \& Sarmiento, J.P. (2020). A Neglected Issue: Informal Settlements, Urban Development, and Disaster Risk Reduction in Latin America and the Caribbean. Disaster Prevention and Management: An International Journal. https://doi.org/10.1108/DPM-04-2020-0115

Sarmiento J.P. (2015). The Ebola Case \& the New Disaster Risk Perspectives. Presented at the UK-US Social Responses to Emergencies. Coral Gables, FL: University of Miami Press.

Wilkinson, A. (2020). Local response in health emergencies: Key considerations for addressing the COVID-19 pandemic in informal urban settlements. Environment and Urbanization, 0956247820922843. https://doi.org/10.1177/0956247820922843

\section{AUTORES}

Adriana Allen. Profesora Titular en Planificación del Desarrollo y Sustentabilidad Urbana en la Bartlett Development Planning Unit (DPU) (University College London, Reino Unido) y Presidenta de la Coalición Internacional del Habitat (HIC). En los últimos 35 años, como académica y activista, la Profesora Allen ha trabajado en más de 25 países del Sur Global, en Asia, África, y América Latina. Su trabajo se ha centrado en el análisis de la interfaz entre las prácticas cotidianas de hacer-ciudad de hombres y mujeres, y las intervenciones planificadas por parte los gobiernos locales, buscando espacios de transformación para generar ciudades, relaciones sociales y medioambientalmente justas. Sus más recientes libros incluyen: Untamed Urbanisms (2016), Environmental Justice and Resilience in the Global South (2017), Urban Water Trajectories (2017), Handbook of Urban Global Health (2019), and Routledge Handbook of Urban Resilience (2020).

Juan Pablo Sarmiento. Médico Cirujano (Universidad del Rosario, Colombia), cuenta con un M.Sc. en Salud Pública, especialidad en Promoción de la Salud y Desarrollo Social (Université de Bordeaux, Francia y Universidad Pública de Navarra, España); un M.A. en Gestión de Proyectos ( UCI, Costa Rica); y una Especialización en Educación Médica (Universidad de la Sabana, Colombia). Tiene estudios de posgrado en Gestión de Desastres (Oxford, Reino Unido); Alta Gerencia Pública (Escuela Superior de Administración Pública, Colombia), residencia en Nutrición (Tufts University, U.S.A.). Actuallmente es profesor investigador y director asociado de investigación en el Instituto de Eventos Extremos de Florida International University. También es director del Programa de Riesgo y Resiliencia en las Américas, financiado por la Agencia de los Estados Unidos para el Desarrollo Internacional (USAID). Publicaciones recientes: https://works.bepress.com/juan-sarmiento/.

Vicente Sandoval. Investigador postdoctoral del proyecto Gobernanza de la Producción y Captación de Conocimientos y Tecnologías Geo-Científicas para la Gestión del Riesgo de Desastres en Indonesia y Chile en el Disaster Research Unit, Freie Universität Berlin, Alemania. Doctor (Ph.D.) en Planificación del Desarrollo (University College London, Reino Unido), Máster (M.Sc.) en Gestión Urbana (TUBerlin, Alemania), y Licenciado en Diseño (UC-Temuco, Chile). Su experiencia es principalmente como investigador de los procesos de desarrollo urbano y desarrollo/cooperación internacional, con foco en la gobernanza urbana del riesgo de desastre, e informalidad urbana y riesgos en Latinoamérica. Ha trabajado además como investigador y consultor internacional para proyectos con la Comisión Europea, GIZ, y USAID. Desde el 2016, colabora como editor de la Revista de Estudios Latinoamericanos sobre Reducción del Riesgo de Desastres (REDER). Publicaciones recientes: http://www.polsoz.fu-berlin.de/kfs/...Sandoval/index.html 